\title{
OPPNets and Rural Areas: An Opportunistic Solution for Remote Communications
}

\author{
Manuel Jesús-Azabal $(\mathbb{D}$, Juan Luis Herrera $(\mathbb{D}$, Sergio Laso $\mathbb{D}$, and Jaime Galán-Jiménez $(\mathbb{D}$ \\ Department of Computing and Telematics Systems, University of Extremadura, Avda. de la Universidad S/N. 10003 - Caceres, Spain \\ Correspondence should be addressed to Manuel Jesús-Azabal; manuel@unex.es
}

Received 12 March 2020; Revised 20 March 2020; Accepted 12 December 2020; Published 15 January 2021

Academic Editor: Nathalie Mitton

Copyright (C) 2021 Manuel Jesús-Azabal et al. This is an open access article distributed under the Creative Commons Attribution License, which permits unrestricted use, distribution, and reproduction in any medium, provided the original work is properly cited.

\begin{abstract}
Many rural areas along Spain do not have access to the Internet. Despite the huge spread of technology that has taken place during recent years, some rural districts and isolated villages have a lack of proper communication infrastructures. Moreover, these areas and the connected regions are notably experiencing a technological gap. As a consequence of this, the implementation of technological health solutions becomes impracticable in these zones where demographic conditions are especially particular. Thus, inhabitants over 65 suppose a large portion of such population, and many elderly people live alone at their homes. These circumstances also impact on local businesses which are widely related to the agricultural and livestock industry. Taking into account this situation, this paper proposes a solution based on an opportunistic network algorithm which enables the deployment of technological communication solutions for both elderly healthcare and livestock industrial activities in rural areas. This way, two applications are proposed: a presence detection platform for elderly people who live alone and an analytic performance measurement system for livestock. The algorithm is evaluated considering several simulations under multiple conditions, comparing the delivery probability, latency, and overhead outcomes with other well-known opportunistic routing algorithms. As a result, the proposed solution quadruples the delivery probability of Prophet, which presents the best results among the benchmark solutions and greatly reduces the overhead regarding other solutions such as Epidemic or Prophet. This way, the proposed approach provides a reliable mechanism for the data transmission in these scenarios.
\end{abstract}

\section{Introduction}

Communication technologies have experienced an exponential spread in the last years. The increasing interest of users on the Internet has unleashed a big competition between Internet Service Providers (ISPs) to provide coverage to as many areas as possible. Furthermore, the lowering prices of broadband access have allowed small businesses and homes to have access to the Internet. Nowadays, the percentage of the population in Spain with access to high-speed Internet connectivity has extraordinarily increased up to values of $81 \%$ in 2019 [1]. In Figure 1, the evolution of the percentage of homes with FTTH (Fiber To The Home) coverage in Spain is shown for recent years. These numbers highlight an average increase of $12.98 \%$ per year, which is expected to continue growing in the next decade. The constant expansion of technology has promoted the improvement of infrastructures and the rise of connected places. Nevertheless, these encouraging statistics are not the same in all regions. Territories like Madrid, Barcelona, or the Basque Country possess the highest numbers of connected homes. However, regions like Extremadura, Castile and León, or Galicia constitute the lowest rates and the biggest "shadow surfaces" [1]. These districts, where Internet infrastructures are minimal, are normally rural and isolated areas in which network operators are not interested in deploying their solutions due to the low benefits they acquire. These circumstances aggravate the problem. Furthermore, along the technology gap, rural areas face additional challenges like healthcare.

Demography in rural areas is commonly characterized by a significant percentage of people who are over 65. In this manner, rural regions face the challenge of providing 


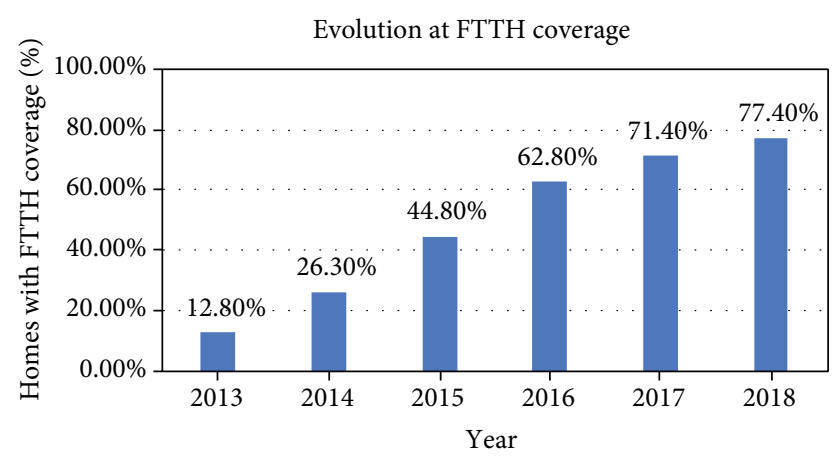

Figure 1: Evolution of FTTH coverage in Spain.

solutions to enhance the lives of elders in this challenging context. Loneliness is one of the main features in elderly people since a meaningful portion of them live alone in their homes. These circumstances can become a severe problem for health, especially in elderly people who are under clinical treatments. As a consequence of the lack of Internet infrastructures, technological solutions to help elderly people in their day-to-day routines cannot be applied, since monitoring systems and telemedicine platforms, which actively depend on the Internet, cannot be deployed. This way, these situations impact on the life of elderly people in rural areas.

On the other hand, technological constraints affect not only the elderly people but the local businesses too. Rural areas are filled with business activities like livestock or agriculture, and they are especially impacted by the conditions of the zone. Technological solutions for industry performance have become disruptive tools in production improvement. These systems provide mechanisms for monitoring and analyzing production processes, being implemented consistently in daily operation. Many of these systems provide a set of tools for production monitoring. In this manner, data processing becomes a relevant feature which requires specific communication infrastructures. In remote rural areas, where there is no Internet access, these procedures cannot be easily deployed.

Taking this context into account, rural areas that lack communication infrastructures face difficult challenges. The specific needs and the technological isolation motivate the spreading of alternative technologies which explore mechanisms for information transmission and system communication. Based on this idea, this paper proposes a DTN-based communication system for healthcare monitorization and livestock data transmission. The proposed platform brings a solution for elderly monitorization without an Internet connection. Thus, presence information about the elderly is transmitted to detect possible dangerous situations derivated from the physical inactivity. As a consequence, mortality rates in elderly people who live alone may decrease, taking into account that emergency detection becomes a crucial factor in healthcare. Moreover, since the platform provides a communication mechanism for isolated areas, local livestock businesses can transmit performance and production data. As a result, the technological gap is addressed while the system provides a healthcare solution for elderly people. The proposed approach exploits the use of SACAR OCVN [2], a routing algorithm based on the cooperation among nodes with different interests. In particular, this algorithm provides the mechanism to forward the information by adapting it depending on the type of data and on the interests of the receiver.

This article proposes the solution structuring the content as follows: firstly, Section 2 consists of a brief study of technological approaches to the communication challenges and rural areas. Section 3 describes our proposal, as well as the system model with the elements composing it. Section 4 shows the experimental results and the algorithm performance, by comparing the obtained results with the outcomes of other well-known opportunistic routing algorithms. Finally, Section 5 draws some conclusions and the next steps in this research line.

\section{Related Work}

During the last years, the research community has been actively working on solutions for communication in isolated areas. These ideas propose an alternative behaviour regarding the classical network operation, using the close proximity between devices and the movement between places. The most popular network technologies based on this idea are Delay Tolerant Networks (DTN). DTN technology is a communication paradigm based on the physical proximity of devices to reach information transmission [3]. This way, low energy interfaces are used to provide communication and broadcast information between the network components or gateways.

DTN are notably deployed in isolated and concrete areas where the Internet connection is not possible. However, the possibilities of DTN are numerous. Hostile-communication contexts like subaquatic communications, spatial transmission, or intermittent connections are some of these opportunities. Moreover, some communication applications do not require a constant information flow like wildlife tracking [4] or low-priority information traffic [5]. This way, DTN provides a suitable option for the deployment of applications and data exchange. Nevertheless, one of the most relevant applications of DTN is the reaction and deployment in natural disaster scenarios. Since the communication architecture is usually critically damaged, DTN provides a useful solution for simple data transmission. The application of DTN in isolated rural areas has led to multiple works in this field. In the early 2000s, many proposals based on DTN brought the connection to remote areas. Projects like Zhang et al. [6] or Pentland et al. [7] are good examples of technology applied in these isolated areas.

In Zhang et al. [6], the authors propose a communication system based on DTN which allows the population at Swedish Lapland to be connected to the Internet. This project addresses the requirement of providing a solution which adapts to the nomadic living conditions of the inhabitants. Following the same line, Pentland et al. [7] brought a lowcost communication infrastructure to remote areas using wireless communications and public transport mobility. Besides, the article includes the successful results of the deployment in isolated parts of India and Cambodia. Both 
proposals are mainly oriented to provide inhabitants with communication services like email or access to documents.

As DTN is improving, recent works explore new prospects. Some projects like Berrocal et al. [8] bring new paradigms like the hybrid model conformed by SDN [9] and DTN, using the movement of the cars and intermediate nodes to relay information to gateways. Other ideas like Galán-Jiménez et al. [10] focus on providing Internet coverage to rural and low-income areas based on $5 \mathrm{G}$ architectures, using Unmanned Aerial Vehicles (UAVs) in remote zones, and exploring optimal energy consumption [11].

Of course, the purpose of these systems is significantly varied. Technological healthcare is especially relevant in rural areas but is arduous to match with the possibilities of DTN. Usually, the healthcare system requires constant communication and Internet connection, factors which are hard to provide using DTN. However, several projects already proposed eHealth solutions using this network paradigm. Works like Galán-Jiménez et al. [12] proposes the use of the DTN model to monitor patients. This specific scheme provides opportunistic communication between the patient and the doctor, using body sensors and smartphones. Moreover, Galán-Jiménez et al. [13] keeps a similar behaviour but benefiting from intermittent connections. As a result, this architecture brings viable ideas which propose a solution for isolation and healthcare in rural areas.

The work presented in this paper is different from the ones introduced above in the sense that technological healthcare is addressed through the integration of SACAR OCVN [2], an opportunistic routing algorithm based on the interests of the nodes and that it is applied in rural areas to provide health monitoring and communication infrastructure for local businesses. Therefore, the proposed model forwards two kinds of information: health info from the ageing inhabitants and production data from smart livestock. Thus, the solution faces the problem of healthcare monitoring through the presence detection of the elderly at home and provides a mechanism to deal with the technological gap in rural industries. In the next section, the behaviour of our proposed algorithm is explained.

\section{OPPNets and Rural Areas}

As previously introduced, this work proposes a solution for healthcare monitoring and data transmission in rural isolated areas. The possibilities of DTN enable the deployment of schemes which allow communication in places where the Internet is not available. Therefore, the provided network scheme benefits from vehicular traffic and physical encounters to transmit two kinds of information: presence data from elderly people's homes and sensor records from livestock. These two types of variables are quite relevant in the proposed scenario.

Presence detection in homes of the elderly is critically relevant. A large number of elderly people live alone at home in isolated rural areas, which becomes a severe health issue in the case of an emergency. This way, the detection of a dangerous situation becomes arduous without human supervision. Nevertheless, there are some relevant clues for possi- ble health risks like interaction within the home environment. Taking this into account, the proposed scheme is based on obtaining data from sensors distributed around the house. These devices provide information about actions like door opening or lights operation. Hence, this presence information is transmitted through the network.

On the other hand, the gateways receive additional information: livestock analytic reports. The usual isolation of many areas of agricultural exploitation and many livestock farms becomes a significant technological gap compared with those businesses which can access the Internet. These industry holdings are especially suitable for the deployment of sensors and production analytics. Nevertheless, the lack of communication infrastructures affects these technological approaches. Thus, using the communication capability of the proposed network, reports from sensors are transmitted into the platform to end gateways.

The network behaviour follows the scheme provided in Figure 2, which identifies several components: (i) sender nodes, (ii) intermediate nodes, and (iii) gateways.

(i) Sender nodes refer to the homes of the elderly people and the smart livestock. These elements generate and transmit new information toward gateway nodes which are connected to the Internet. Thus, the messages are sent into the network in specified intervals of time. This way, the nodes send the messages to reachable intermediate nodes, which serve as data mules

(ii) Intermediate nodes are in charge of forwarding messages to the gateway. Therefore, cars and pedestrians receive, carry, and deliver the information as they move through the streets and roads. These nodes decide the information which they prefer to obtain and broadcast: presence info or livestock performance data. Additionally, there is another fundamental element in the scenario: throwboxes. These devices are placed on the main points of the road path and can store messages from any other intermediate node. Thus, throwboxes work as a "meeting point" for data and distribute it to other interested reachable nodes

(iii) Gateways are the destination elements of the messages generated by the sender nodes. They are connected to the Internet and are in charge of processing and transmitting information to the Cloud. As a result, the collected data can be externally processed, enabling the detection of anomalous patterns in the elderly activity and recognizing a possibly dangerous situation. In the same way, the performance information about livestock can be processed when it is finally delivered

The opportunistic behaviour of the network is mainly based on the routing algorithm we propose to perform distributed communication among the aforementioned elements. In this paper, the idea behind the SACAR OCVN algorithm defined in [2] is applied to the rural scenario in 
OPPNets and rural areas

an opportunistic solution for communications

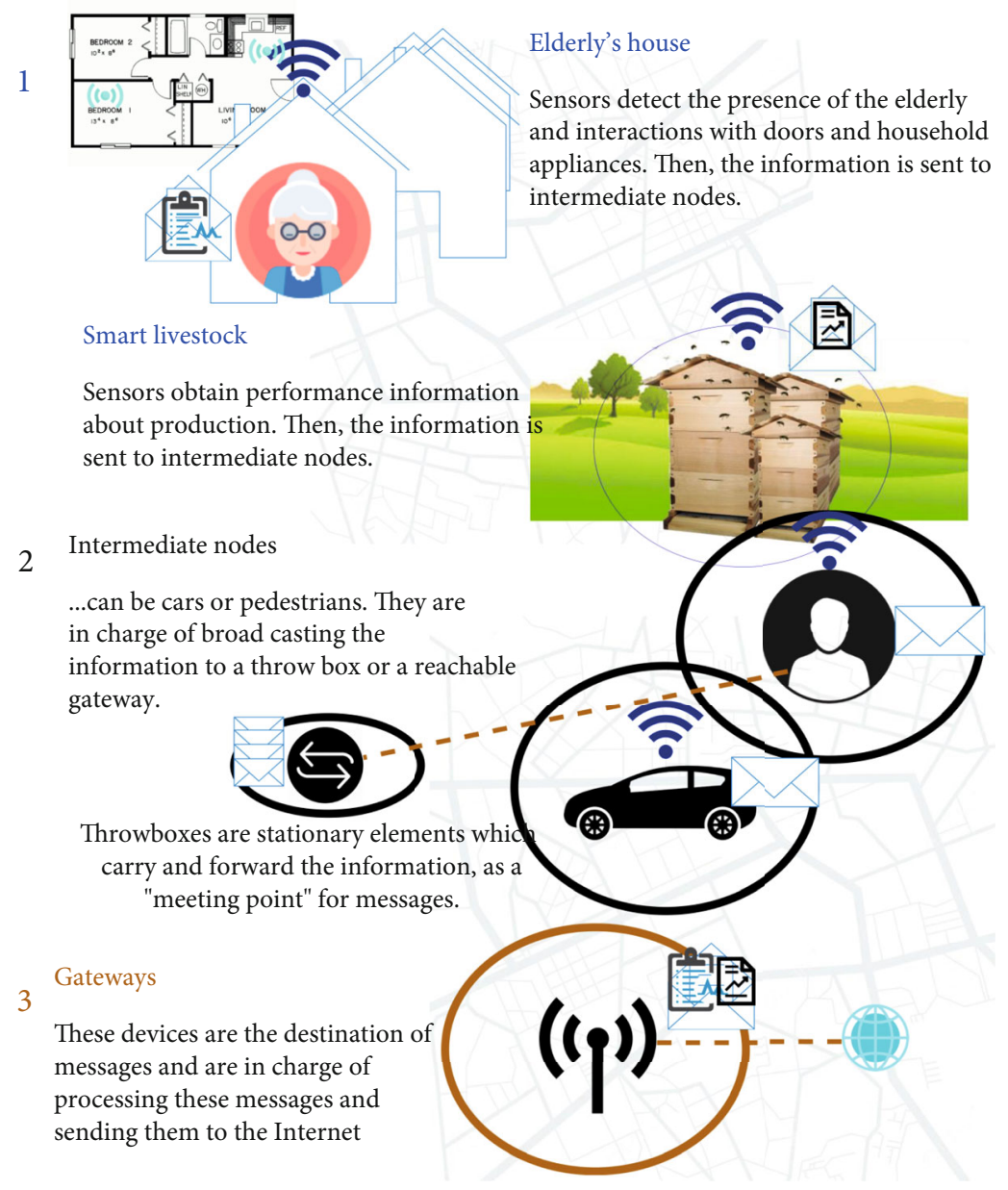

FIGURE 2: Working scheme of OPPNets in rural areas.

order to relay on cooperating nodes to successfully deliver the information from elders and livestock. The behaviour of the nodes in the scenario is based on the preferences of the nodes, following the Situational-Context model [14]. This paradigm proposes an automatic communication scheme for devices, using a virtual profile which defines the node's capabilities and preferences.

SACAR OCVN [2] applies the Situational-Context scheme as a means to automatise the nodes' encounter process. Thus, the nodes in the scenario have a virtual profile which identifies them as sender nodes, intermediate nodes, or gateways. The different roles available on the virtual profile are Goals and Skills: the first one defines the information preferences, while the Skills indicate the actions the node is able to perform. This model, once it is adapted to the scenario, is used to distinguish between sender elements (represented in Figure 3), carrying nodes (depicted in Figure 4), and end gateways (Figure 5).

The communication takes place when two nodes encounter each other. This way, by using low-energy technologies, the information is exchanged within the corresponding range. Thus, the communication process follows three main steps: (1) devices encounter each other, (2) virtual profiles

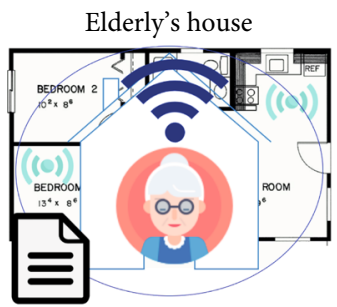

Goals

PresenceReport

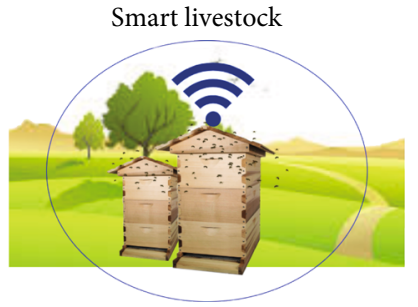

Goals ProductionReport
FIgURE 3: Virtual profile of sender nodes.

are gathered, and (3) information is exchanged. All these steps are shown in Figure 6. The proposed scenario raises an opportunistic context where communication provides an improvement in the day-to-day routines of local inhabitants. The idea has been executed as a simulation which has tested the algorithm under different situations, trying to define the most appropriate context for interactions. The next section details the simulation process and the parameters that have been tuned. 

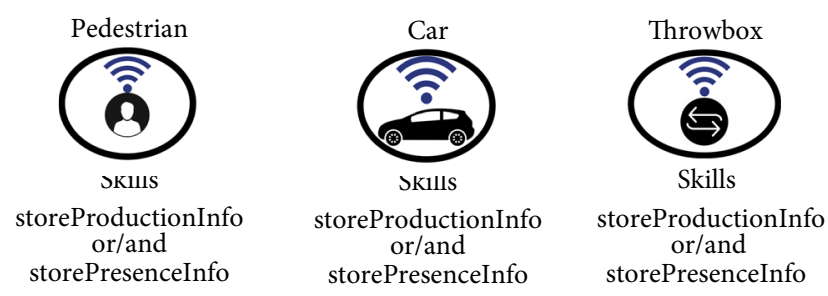

FIGURE 4: Virtual profile of intermediate nodes.

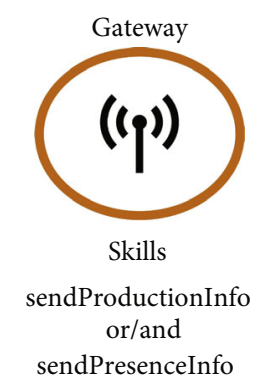

FigURE 5: Virtual profile of gateway nodes.

\section{Experimental Results}

The result section is organized as follows: firstly, the selected scenario is described. Then, the results are analyzed focusing on two aspects: (i) performance analysis of SACAR OCVN algorithm on the scenario and (ii) comparison with other relevant opportunistic routing solutions.

4.1. Simulated Scenario. Simulations have been carried out using The ONE simulator [15]. This tool provides a development environment for DTNs, allowing the specification of a detailed scenario, movements, and protocols. Thus, the performance of the routing algorithm can be obtained through execution reports. In this work, the simulated scenario recreates a rural area with low connectivity, which reflects a village community and the surrounding roads. Figure 7 represents the simulation map.

The simulation process follows an execution scheme which varies the context inputs and node behaviour. Table 1 summarizes the input configuration. The number of total nodes is $N=146$, where $N_{s}=3$ is the number of destination nodes, $N_{i}=66$ is the number of intermediate nodes, and $N_{g}=80$ are referred to sender nodes. Destination locations have been established far away from the rural village in order to analyze the effectiveness of the communication. Intermediate nodes are distributed along paths in an area of $A=81000 \mathrm{~m}^{2}$ with different movements patterns, $P$, which specify how the nodes move. The type of interest settings is composed of the Skill set, $S$, and the Goal set, $G$. The first group keeps the capabilities of the nodes, in this case, store-and-carry presence information or performance data. Also, the Skill of transmitting messages to the Internet is possible. On the other hand, the Goal set defines, in this case, the kind of information the node generates. The maximum number of Goals in the nodes is $g_{n}=2$ and $s_{n}=2$ in the case of Skills. Simulation duration is set to $T=28000 \mathrm{~s}$.

Message generation interval, $\omega$, is in charge of establishing the waiting time before the sender nodes generate new information. It is a key parameter in the simulations since the number of created messages depends actively on it. In this scenario, three times are considered: $\omega=\{900,1800,3600\}$ s. These intervals adapt the message generation to real values since presence data requires a fluent transmission. In the next section, simulation results are analysed, as well as compared with other well-known opportunistic routing algorithms under different contexts.

4.2. Parameter Setting. Four main outcomes are analyzed after running the simulations: $(1)$ delivery probability $\left(d_{\text {prob }}\right)$, (2) overhead ratio $(\theta)$, (3) average latency $(\tau)$, and (4) average number of hops $(\gamma)$ :

(i) $d_{\text {prob }}$ is the percentage of messages which reached the destination node. This way, it is a critical value which reflects the success of communication and forwarding. This value is calculated as the relation between the number of messages originally sent, $d_{\text {sent }}$, and the number of messages received at destinations, $d_{\text {received }}$

(ii) $\theta$ is the relation between duplicated messages and received messages. It reflects the use of the network

(iii) $\tau$ reflects the average time needed to receive a message once it is sent

(iv) $\gamma$ represents the average number of intermediate nodes needed to reach the destination

These results are obtained when the simulation process is finished. This way, the scenario receives two main inputs which vary the final results. These two variables are interval message generation $(\omega)$ and interest distribution at intermediate nodes.

(1) Interval message generation, $\omega$, specifies the waiting time before new messages are created. Thus, it defines a variable which plays an important role in simulation. The considered times are 900, 1800, and 3600 seconds

(2) Since SACAR OCVN is based on node interests, three different distributions of interests are considered: (i) the percentage of nodes only interested in carrying elderly presence information $\left(I_{\text {elderly }}\right)$, (ii) nodes only interested in carrying industrial production information (e.g., from livestock) $\left(I_{\text {industry }}\right)$, and (iii) nodes interested in carrying both types of information $\left(I_{\text {hybrid }}\right)$. Moreover, there are also nodes which are not interested in carrying any type of information $\left(I_{\text {empty }}\right)$. Table 2 shows the selected values for the three considered scenarios 


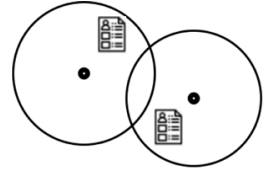

1. device makes contact

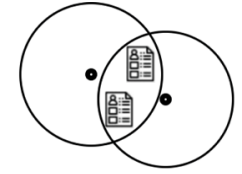

2. virtual profilesare read

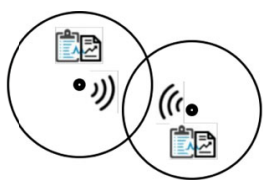

3. information exchange

FIgURE 6: Detailed communication process.

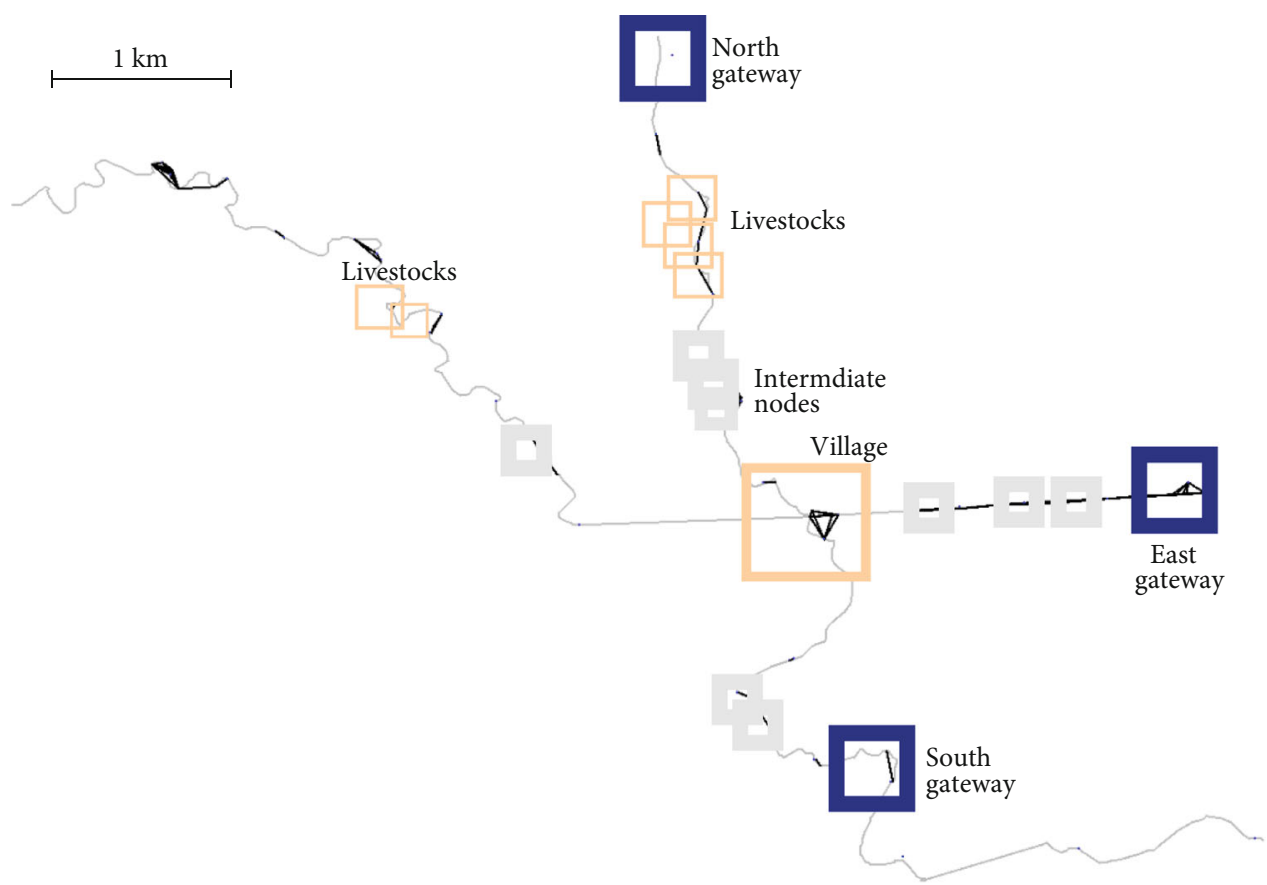

Sender nodes

Gateway nodes

Intermediate nodes

FIgURE 7: Rural scenario simulated in The ONE.

TABLE 1: Parameter setting for the rural scenario.

\begin{tabular}{lc}
\hline Parameter & Value \\
\hline$A$ & $81000\left[\mathrm{~m}^{2}\right]$ \\
$N$ & 146 \\
$N_{s}$ & 3 \\
$N_{i}$ & 66 \\
$N_{g}$ & 80 \\
$S$ & StoragePresenceInfo, StorageProductionInfo $\}$ \\
$G$ & $\{$ PresenceReport, ProductionReport\} \\
$s_{n}$ & 2 \\
$g_{n}$ & 2 \\
$P$ & $\left\{\right.$ StationaryMovement for $N_{s}$, StationaryMovement for \\
$P$ & $N_{g}$, MapRouteMovement for $N_{i}$ with stationary time \\
$T$ & periods in the range of $t=[300,500]$ s. $\}$ \\
$\omega$ & $28000[\mathrm{~s}]$ \\
\hline
\end{tabular}

TABlE 2: Percentage of nodes contemplated for scenario simulations.

\begin{tabular}{lcccc}
\hline Scenario & $\begin{array}{c}I_{\text {elderly }} \\
(\%)\end{array}$ & $\begin{array}{c}I_{\text {industry }} \\
(\%)\end{array}$ & $\begin{array}{c}I_{\text {hybrid }} \\
(\%)\end{array}$ & $\begin{array}{c}I_{\text {empty }} \\
(\%)\end{array}$ \\
\hline $\begin{array}{l}\text { Elderly interest } \\
\text { scenario }\end{array}$ & 50 & 20 & 20 & 10 \\
$\begin{array}{l}\text { Factory interest } \\
\text { scenario }\end{array}$ & 20 & 50 & 20 & 10 \\
$\begin{array}{l}\text { Hybrid interest } \\
\text { scenario }\end{array}$ & 35 & 35 & 20 & 10 \\
\hline
\end{tabular}

\section{SACAR OCVN Performance Analysis}

The proposed algorithm is executed on each scenario distribution, varying the message generation interval, $\omega$. As Figure 8 reflects, the delivery probability $\left(d_{\text {prob }}\right)$ obtained by SACAR OCVN is very relevant. Since it is a critical value which captures the success at message transmission, a high percentage is needed. This way, the algorithm experiences the lowest rates when the message generation interval is low. On the other hand, the best results are obtained when 


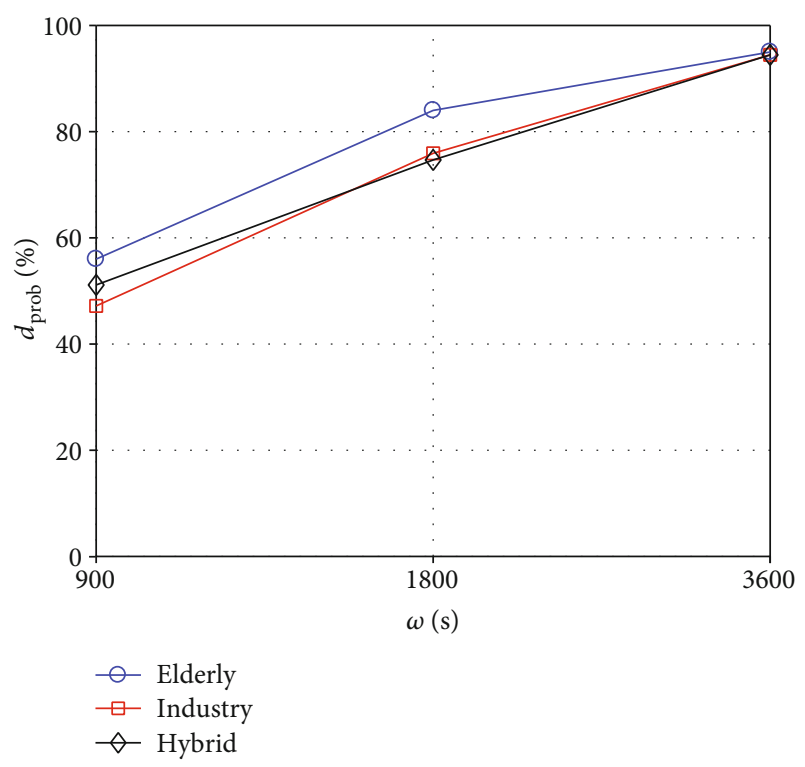

FIGURE 8: Delivery probability in SACAR OCVN.

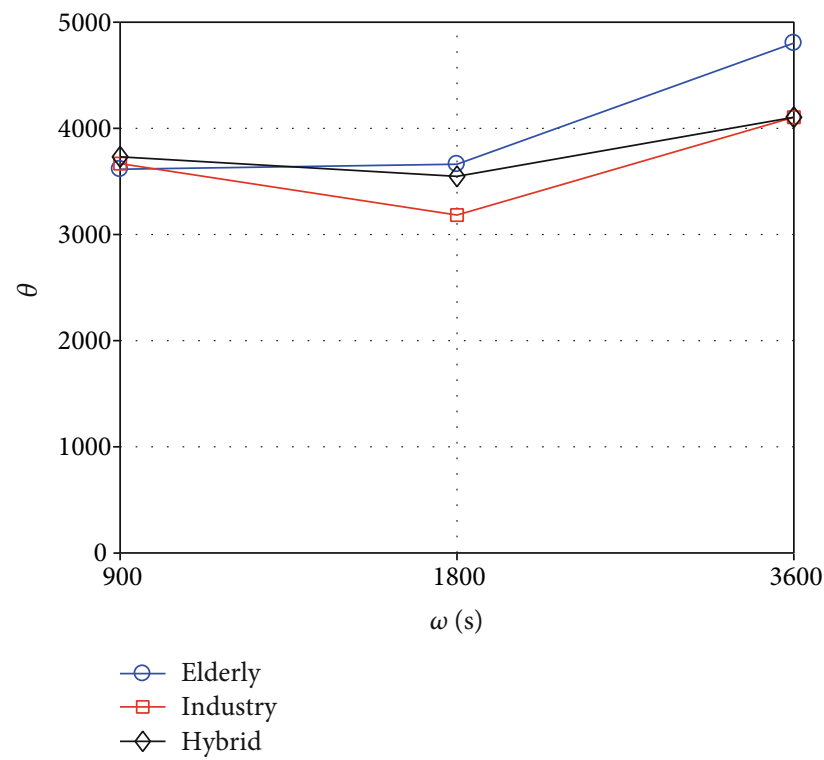

FIGURE 9: Overhead ratio in SACAR OCVN.

the value is increased, reaching $d_{\text {prob }}=0.945$ when $\omega=3600 \mathrm{~s}$ . This is essentially due to low interval values which involve a larger number of messages to be transmitted.

The overhead ratio results are included in Figure 9. This value is a suitable parameter to know the practical usage of the bandwidth in the network. SACAR OCVN experiences an overhead increase when the predominant interest is the information from elderly people. On the other hand, when industry interest is predominant, the value of $\theta$ decreases. As a result, the algorithm experiences an average result when the scenario is mixed.

Since the transmitted information is elderly-care related, the average latency $(\tau)$ is critical to minimize the needed time to reach the destination. As Figure 10 shows, SACAR OCVN

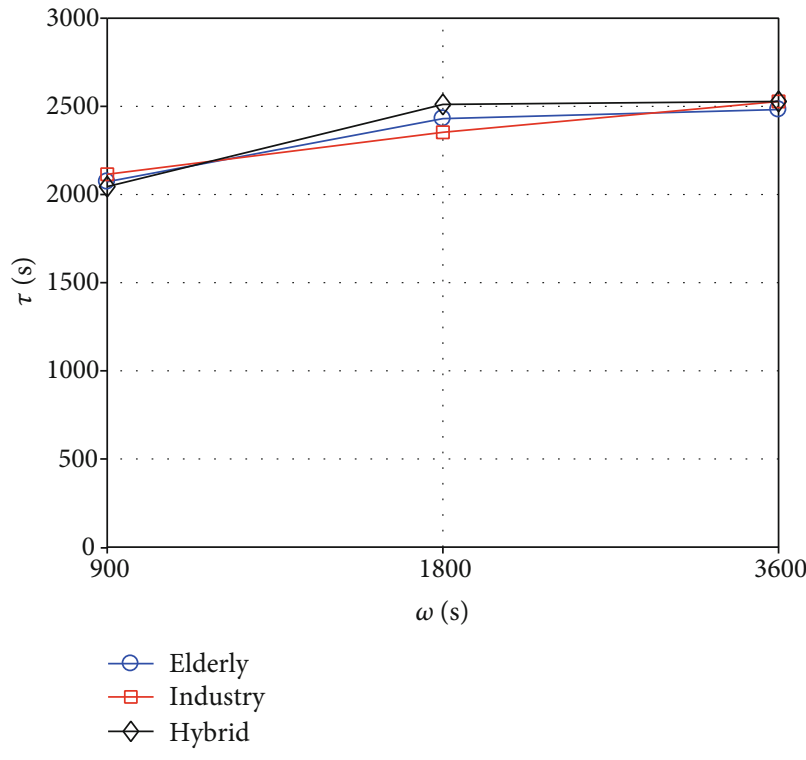

FIgure 10: Average latency in SACAR OCVN.

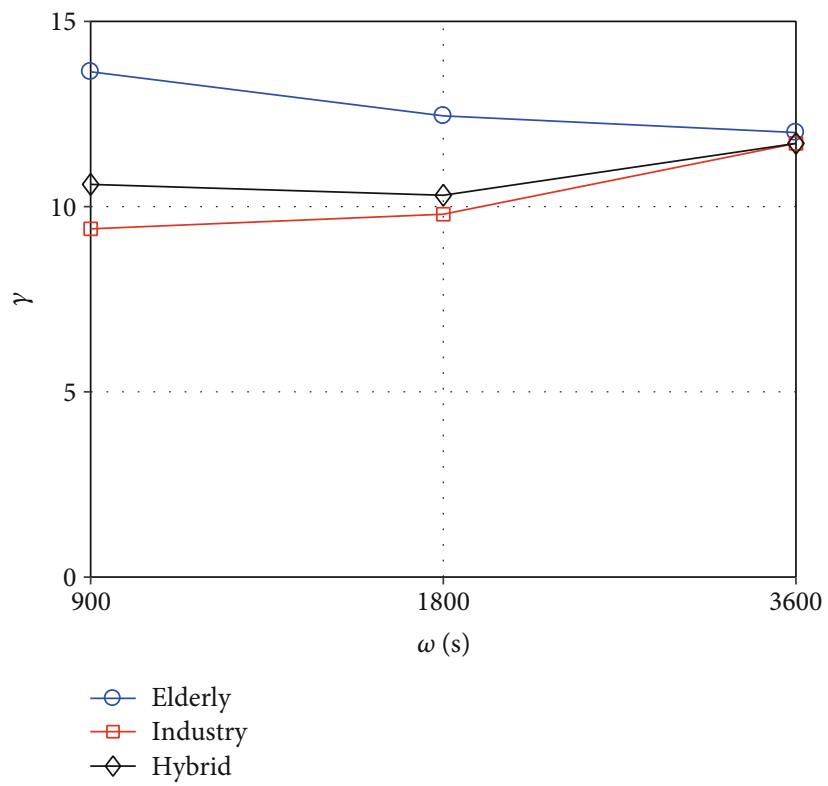

Figure 11: Average hops in SACAR OCVN.

presents very similar results in each scenario with an average latency of around $\tau=2500 \mathrm{~s}$.

Finally, the average number of hops, $\gamma$, is the median number of intermediate nodes needed by the messages to reach the destination. The latency value is usually related to the hop rate since the encounters and information transmission at hops improve possibilities of delivery. This way, as Figure 11 shows, SACAR OCVN average hop outcomes follow an inversely proportional trend w.r.t. latency values.

As a conclusion, SACAR OCVN results are positive. The delivery probability in $\omega=3600 \mathrm{~s}$ is $d_{\text {prob }}=0.945$, guaranteeing a high rate at message broadcasting. Besides, the message generation interval which provides a better performance is $\omega=3600 \mathrm{~s}$. This successful result means that the algorithm 
provides a good communication mechanism for the exposed scenario. As a consequence, the data of presence from the elderly's homes is fluently transmitted through the network. Thus, the system allows the remote detection of possible dangerous situations, derivated from the inactivity of the elderly. Besides, the local industries can transmit performance data from the exploitation.

Once SACAR OCVN best results are obtained, they are compared under the same context conditions with the rest of the DTN algorithms included in The ONE. Furthermore, this second stage of result analysis is addressed.

\section{Comparison with Other Opportunistic Routing Solutions}

SACAR OCVN experiences better results when the message generation interval is $\omega=3600 \mathrm{~s}$. This way, using the same scenario conditions, in this section, we compare these values with the ones obtained when running other well-known opportunistic routing algorithms in The ONE. Note that the analysis uses the same set of parameters described in the previous section. Next, benchmark algorithms are briefly described. DirectDeliveryRouter [16] works based on straight delivery between the sender and the receiver node. Thus, intermediate elements are ignored. EpidemicRouter [17], on the other hand, belongs to the flood algorithm family. Thus, the behaviour is based on duplicating the messages with every encountered node. MaxPropRouter [18] uses the previous node encounters to define the most appropriate path to the destination. ProphetRouter [19] works based on a probabilistic scheme while SprayAndWaitRouter [20] replies to messages by creating copies which can be specified by the user.

The executions take place with $\omega=3600 \mathrm{~s}$ for the different interest distributions over the same scenario described in Figure 7. Delivery probability is the most significant parameter in performance comparison. As Figure 12 shows, the best delivery probability is $d_{\text {prob }}=0.95$ and belongs to the SACAR OCVN execution. The other algorithms provide a low delivery rate in the three scenarios.

Overhead ratio results are reflected in Figure 13. DirectDeliveryRouter is based on communicating sender nodes and destination, without using intermediate elements. Thus, message traffic is low. Besides, the lowest values belong to SprayAndWaitRouter, which provides the communication process with better use of the bandwidth than EpidemicRouter and ProphetRouter. SACAR OCVN, in turn, keeps close values to SprayAndWait.

Figure 14 shows the average latency in executions. SACAR OCVN is the algorithm which has the highest latency value, mainly because of the largely supported message traffic, as well as the low hop rates. On the other hand, EpidemicRouter and ProphetRouter keep the results below these numbers. Besides, SprayAndWaitRouter provides the lowest rate.

The average number of hops in each of the algorithms is presented in Figure 15. DirectDeliveryRouter bases its behaviour on senders delivering messages straight to the destina-

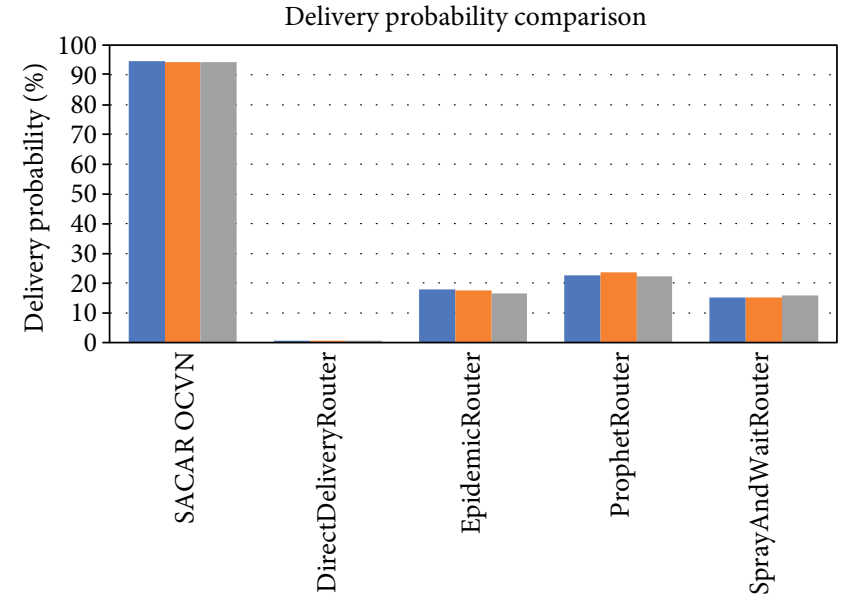

Routing algorithm

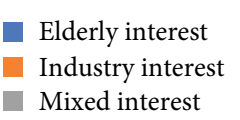

Figure 12: Delivery probability comparison.

tion; thus, it is always one hop. On the other hand, SACAR OCVN keeps an average hop number quite low regarding EpidemicRouter and ProphetRouter results, which represent the highest in the hybrid interest scenario. Again, SprayAndWaitRouter provides a good performance and draws on few hops to reach the destination.

The comparison process clearly shows the performance of SACAR OCVN compared with the opportunistic routing algorithms included in The ONE. The high delivery probability value guarantees the applicability of the solution in the described scenario, becoming a powerful tool in the implementation of the project. It is important to remark that SprayAndWaitRouter keeps good results at $\theta, \tau$, and $\gamma$, but $d_{\text {prob }}$ is too low to provide a reliable communication. The possibilities of the router are relevant and follow a promising work line.

\section{Discussion}

Rural areas represent a big percentage of the population over 65. Only in Spain, around 30\% of inhabitants in small villages are older adults. These demographic conditions become a challenge for health, since issues like personal assistance, solitude, and isolation play a big role in the daily routines of seniors. Technology has become a very significant ally for healthcare, providing mechanisms and tools such as eHealth, remote monitoring, and smart systems which ease communication and quick emergency detection. However, many of these rural spaces lack Internet connection, where no infrastructures to deploy eHealth solutions can be exploited.

In this paper, a response to this context is provided. Making use of alternative communication mechanisms like opportunistic networks, a reliable system for remote areas is introduced. Thus, the proposal supplies villages with an architecture for message transmission which allows the detection of possible emergencies of seniors who live alone 


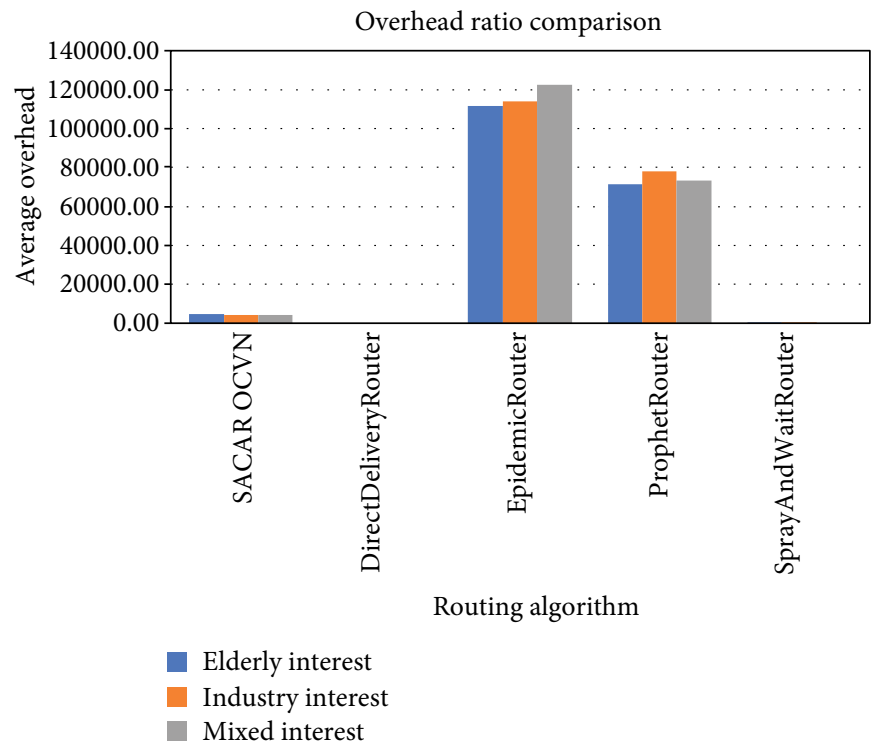

FIGURE 13: Overhead ratio comparison.

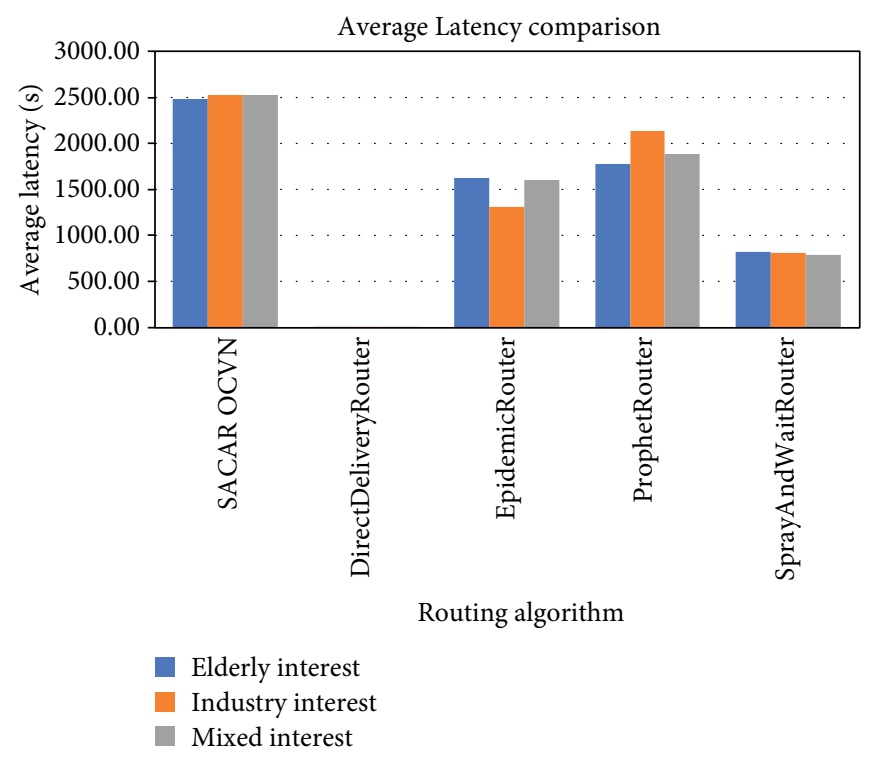

FIGURE 14: Average latency comparison.

while local exploitations are also able to transmit performance reports.

On the one hand, information about the presence habits of seniors who live alone is transmitted. This way, several sensors are installed along the houses, recording the presence of the elderly and sending such information toward the opportunistic network. The possibility of determining the presence patterns of elderly people allows the detection of potentially risky situations that can happen in the solitude of the home. As a result, mortality risks in the elderly who live alone are reduced by exploiting the detection of anomalous behaviour.

On the other hand, the local exploitations in isolated rural areas face the challenge of the lack of Internet infrastructure. The technological gap reduces the competitive advantages compared to the connected industries, and therefore, their maximum performance capabilities cannot be reached. In order to tackle this problem, the proposed solution exploits the use of sensors at the exploitation place like livestocks or hives, playing roles like weight measurement, animal tracking, or food consumption.

The data collected by sensors is broadcasted toward the destination making use of BLE technology and intermediate nodes. These nodes, which serve as mules, can be different types of devices like cars, smartphones, smartwatches, or throwboxes. These lasts are devices installed on the roads, fed by solar panels, which store and forward the information to the devices in their range.

As a result, the proposed solution in this paper is aimed at improving the quality of life in remote areas. 


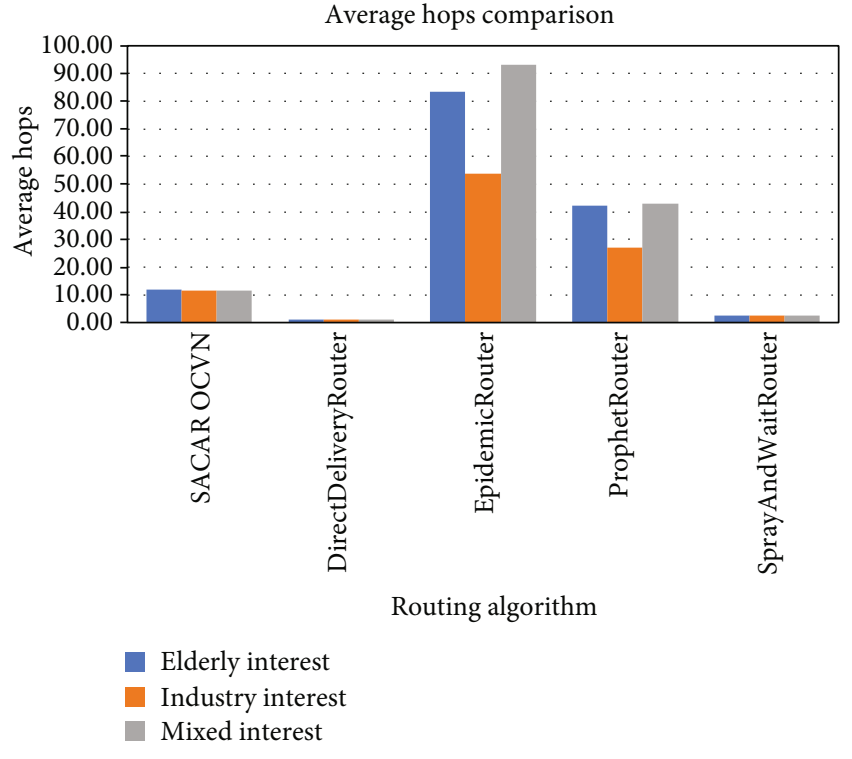

FIGURE 15: Average hops comparison.

Communication possibilities in these scenarios go further and provide an eHealth architecture for eldercare and local business improvement. In this way, the opportunities of deploying a prototype in a real isolated zone could bring a brand new autonomous model for well-being, alliance, and cooperation.

\section{Conclusions and Future Works}

Many isolated rural areas do not have an Internet connection. These limitations are often induced by the remote situation and the geographical issues, as well as the lack of interest from telecommunication companies. Thus, inhabitants cannot keep connected in a world where the Internet expansion is exponentially growing. Furthermore, the population in rural areas is usually over 65 years old. Thus, isolated regions face the difficult challenge of providing services and health attention, especially when these elderly people live alone. However, this context can be improved by using technological solutions. Digital elder healthcare is an active work line where many researchers and companies are working on. Nevertheless, the lack of an Internet infrastructure prevents the deployment of many of these solutions. Moreover, the technological gap also affects the local industry and livestock, which are not generally able to implement solutions to monitor and improve performance.

In order to face these challenges, DTN solutions become a suitable technology to provide connectivity in remote places. This paper proposes a DTN routing algorithm based on information interests which transfer elderly presence information and industry performance data to gateway points.

Our proposed algorithm has been simulated on a realistic isolated region of Spain. The obtained results are positive and guarantee a high rate of successful message delivery under different conditions. Furthermore, the outcomes of our proposed algorithm are also compared with other well-known opportunistic routing solutions, outperforming them. Therefore, the proposed scheme brings a solution to the technological gap in isolated rural areas, enabling the monitoring of elders' activity and providing a reliable communication system to improve the performance of livestock industries.

The authors are working on providing the proposed algorithm with a smart behaviour through a machine learning model. Moreover, trajectory prediction in nodes' movement presents other key aspects to be explored.

\section{Data Availability}

Data are available on request. Research results are available on request. Thus, in order to be provided with the data report, please, write to Manuel Jesús-Azabal (manuel@unex.es).

\section{Conflicts of Interest}

The authors declare that they have no conflicts of interest.

\section{Acknowledgments}

This work was supported by 4IE+ project (0499_4IE_PLUS_ 4_E) funded by the Interreg V-A Espańa-Portugal (POCTEP) 2014-2020 program, by RTI2018-094591-B-I00 Project (MCI/AEI/FEDER,UE), by the Department of Economy, Science and Digital Agenda of the Government of Extremadura (GR18112, IB18030), and by the European Regional Development Fund.

\section{References}

[1] J. Burgess, B. Gallagher, D. D. Jensen, and B. N. Levine, "Maxprop: routing for vehicle-based disruption-tolerant networks," in Proceedings IEEE INFOCOM 2006. 25TH IEEE International Conference on Computer Communications, pp. 1-11, Barcelona, Catalunya, Spain, 2006.

[2] A. Lindgren, A. Doria, and O. Schelén, "Probabilistic routing in intermittently connected networks," SIGMOBILE Mob. Comput. Commun. Rev, vol. 7, no. 3, pp. 19-20, 2003.

[3] N. McKeown, T. Anderson, H. Balakrishnan et al., "OpenFlow," ACM SIGCOMM Computer Communication Review, vol. 38, no. 2, pp. 69-74, 2008.

[4] T. Spyropoulos, K. Psounis, and C. S. Raghavendra, "Spray and wait: an efficient routing scheme for intermittently connected mobile networks," in Proceedings of the 2005 ACM SIGCOMM Workshop on Delay-Tolerant Networking, WDTN, vol. 5, pp. 252-259, New York, NY, USA, 2005, ACM..

[5] A. Vahdat and D. Becker, Epidemic Routing for PartiallyConnected Ad Hoc Networks, Duke University Durham, Technical report, 2000.

[6] P. Zhang, C. M. Sadler, S. A. Lyon, and M. Martonosi, "Hardware design experiences in zebranet," in Proceedings of the $2 \mathrm{Nd}$ International Conference on Embedded Networked Sensor Systems, SenSys, vol. 4, pp. 227-238, New York, NY, USA, 2004, ACM.

[7] L. Amorosi, L. Chiaraviglio, and J. Galán-Jiménez, "Optimal energy management of UAV-based cellular networks powered by solar panels and batteries: formulation and solutions," IEEE Access, vol. 7, pp. 53698-53717, 2019. 
[8] J. Berrocal, J. Garcia-Alonso, C. Canal, and J. M. Murillo, "Situational-context: a unified view of everything involved at a particular situation," in International Conference on Web Engineering, pp. 476-483, Springer, 2016.

[9] E. Borgia, R. Bruno, and A. Passarella, "Making opportunistic networks in IoT environments CCN-ready: a performance evaluation of the mobccn protocol," Computer Communications, vol. 123, pp. 81-96, 2018.

[10] Ministerio de Asuntos Económicos y Transformación Digital (es), "El 81\% de la población española dispone de cobertura de Internet a más de 100 Mbps," October 2020 https://cutt .ly/YgmAT2N.

[11] A. Doria, M. Uden, and D. P. Pandey, "Providing connectivity to the saami nomadic community," International Conference on Open Collaborative Design for Sustainable Innovation, vol. 1, 2002.

[12] J. Galán-Jiménez, J. Berrocal, J. Garcia-Alonso, and M. J. Azabal, "A novel routing scheme for creating opportunistic contextvirtual networks in IoT scenarios," Sensors, vol. 19, no. 8, p. 1875, 2019.

[13] J. Galán-Jiménez, L. Chiaraviglio, L. Amorosi, and N. BlefariMelazzi, "Multi-period mission planning of UAVs for 5G coverage in rural areas: a heuristic approach," in 2018 9th International Conference on the Network of the Future (NOF), pp. 5259, IEEE, Poznań, Poland, 2018.

[14] P. Juang, H. Oki, Y. Wang, M. Martonosi, L. S. Peh, and D. Rubenstein, "Energy-efficient computing for wildlife tracking: design tradeoffs and early experiences with zebranet," in Proceedings of the 10th international conference on Architectural support for programming languages and operating systems, pp. 96-107, San Jose, California, USA, 2002.

[15] E. Max-Onakpoya, A. Jacobs, and C. E. Baker, "An opportunistic mhealth architecture for remote patient monitoring," in Proceedings of the 20th International Workshop on Mobile Computing Systems and Applications, pp. 169-169, Santa Cruz, CA, USA, 2019.

[16] E. Max-Onakpoya, S. Madamori, and C. E. Baker, "Utilizing opportunistic social networks for remote patient monitoring in rural areas," in Proceedings of the 1st ACM International Workshop on Technology Enablers and Innovative Applications for Smart Cities and Communities, pp. 46-49, New York, NY, USA, 2019.

[17] A. Pentland, R. Fletcher, and A. Hasson, "Daknet: rethinking connectivity in developing nations," Computer, vol. 37, no. 1, pp. 78-83, 2004.

[18] K. Shin, K. Kim, and S. Kim, "Traffic management strategy for delay-tolerant networks," Journal of Network and Computer Applications, vol. 35, no. 6, pp. 1762-1770, 2012.

[19] Y. Teranishi, T. Kimata, E. Kawai, and H. Harai, "Hybrid cellular-DTN for vehicle volume data collection in rural areas," in 2019 IEEE 43rd Annual Computer Software and Applications Conference (COMPSAC), vol. 1, pp. 276-284, Milwaukee, Wisconsin, USA, 2019.

[20] A. Keränen, J. Ott, and T. Kärkkäinen, "The ONE simulator for DTN protocol evaluation," in SIMUTools '09: Proceedings of the 2nd International Conference on Simulation Tools and Techniques, New York, NY, USA, 2009ICST. 